\title{
Nuevas rutas de permeabilidad en Plasmodium falciparum: consideraciones como blanco farmacológico y avances de los potenciales inhibidores
}

\author{
New permeability pathways in Plasmodium falciparum: considerations as a pharmacological \\ target and advances in potential inhibitors
}

Yeison Castañeda-Agudelo ${ }^{1 *}$, Adriana Pabón-Vidal ${ }^{1}$

\section{Resumen}

Con base en la persistencia de los casos de malaria, dados por múltiples razones, entre las cuales se encuentra la resistencia de Plasmodium falciparum a los antimálaricos establecidos para su tratamiento, surge la necesidad de encontrar nuevos compuestos antimaláricos frente a otros blancos terapéuticos. En vista de ello, las Nuevas Rutas de Permeabilidad (NPP) han sido un objetivo promisorio y a partir de su descubrimiento, se han realizado diversos estudios enfocados en comprender su mecanismo y los componentes utilizados para el transporte de solutos y otras moléculas a través de la membrana del eritrocito. El objetivo de esta revisión de tema es presentar una recopilación de los estudios más significativos realizados en torno a las NPP. Para ello, se revisó la literatura para conocer el estado del desarrollo de las investigaciones referentes al tema, consultando bases de datos electrónicas y combinando los descriptores o palabras clave: (NPP AND malaria, AND Plasmodium falciparum AND inhibitors), además, se realizaron búsquedas secundarias en las listas de referencias bibliográficas de los artículos identificados. Luego de revisar los artículos de la literatura publicados entre los años 1980 y 2019, se concluye que muchos estudios han sido dedicados a la búsqueda de inhibidores de esta vía con fines terapéuticos y hay gran cantidad de moléculas candidatas como el híbrido MA-DFO y las diaminidas que han mostrado actividad en modelos de malaria tanto in vitro como in vivo.

Palabras clave: NPP, Plasmodium, malaria, antimaláricos, eritrocito

\begin{abstract}
In malaria, the persistent resistance to antimalarial drugs by Plasmodium falciparum constitute a problem to world public health. New Permeability Pathways (NPP) are a promissory therapeutic target, because the parasite uses them to modify the membrane of the erythrocyte and make it permeable to various nutrients needed for its growth. We present a theme review where a compilation of various studies related to NPP, its importance and its inhibitors was made.
\end{abstract}

Keywords: NPP, Plasmodium, malaria, antimalarials, erythrocyte

\footnotetext{
1. Grupo Malaria, Universidad de Antioquia, Medellín, Colombia.

* Autor para correspondencia: <yeison.castanedaa@udea.edu.co>
} 


\section{INTRODUCCIÓN}

La malaria es una enfermedad causada por parásitos unicelulares del género Plasmodium; cinco especies son infectivas de forma natural para el hombre: $P$. falciparum, $P$. vivax, $P$. malariae, $P$. ovale y $P$. knowlesi. Esta enfermedad representa un gran problema de salud pública a nivel mundial debido a su alto rango de morbimortalidad, presentando aproximadamente 229 millones de casos en 2019 y 409.000 muertes estimadas para el mismo año, de las cuales la gran mayoría se presentaron en el continente africano (aportando aproximadamente un $93 \%$ de las muertes) (WHO, 2020). En Colombia, entre la semana 1 y la semana 52 del año 2019, se presentaron 78.513 casos de malaria, entre los cuales 1341 corresponden a malaria complicada. De las infecciones presentadas en el país, $P$. falciparum tuvo una prevalencia de $50,3 \%$ aproximadamente de los casos, seguido por $P$. vivax con un $48,6 \%$, y $1,1 \%$ de infecciones mixtas (INS, 2019).

Plasmodium posee un ciclo de vida complejo (Bannister y Mitchell, 2003) (figura 1), que involucra una fase asexual en el humano, invadiendo inicialmente hepatocitos y posteriormente pasando a sangre periférica, donde continúa su ciclo asexual o se diferencia en gametocitos, los cuales son la forma infectiva para el mosquito hembra del género Anopheles en donde sucede la fase sexual (Prudêncio et al., 2006). En la actualidad se presenta una situación crítica frente al tratamiento para la malaria, debido a que los parásitos están presentando resistencia a los medicamentos utilizados; tal como sucede con la cloroquina (CQ), la mefloquina (MQ), la quinina (Q) en $P$. falciparum y la sulfadoxina/pirimetamina (SDXP) mayormente en P. vivax (Chassaigne, 2001; López y Segura, 2008). Recientemente, se identificó resistencia a la Artemisinina y sus derivados (Martin et al., 2018), por lo que es urgente la búsqueda tanto de nuevos compuestos eficaces contra el parásito como de nuevos blancos terapéuticos.

Los eritrocitos son las células blanco de los merozoitos, estadio con capacidad de invasión del ciclo eritrocitario asexual de Plasmodium; allí los parásitos se alimentan de hemoglobina y esta célula les brinda protección contra el sistema inmune. Sin embargo, el parásito necesita, para su correcto desarrollo, nutrientes que no se encuentran en la célula huésped por lo que utiliza la membrana del eritrocito para el transporte de compuestos disponibles en el medio extracelular; para ello, P. falciparum exporta proteínas que amplifican y/o permiten el eflujo e influjo de compuestos pequeños tales como aminoácidos, aniones haluro, cationes orgánicos e inorgánicos $\left(\mathrm{Ca}^{++}\right.$, $\mathrm{K}^{+}$), monosacáridos (sorbitol, manitol) y lactato, entre otros (López y Segura, 2008; Zipprer et al., 2014). A estos cambios en la permeabilidad de la membrana en el eritrocito, dados por el parásito, se les denominaron nueva(s) ruta(s) de permeabilidad (New Permeability Pathways, NPP) (Kirk, 2001).

Gracias a estos hallazgos, se han llevado a cabo esfuerzos para obtener la caracterización profunda de las NPP y establecerlas como un potencial blanco farmacológico (Kirk, 2001). Se registran diversos compuestos con actividad contra las nuevas vías, lo cual resulta ser promisorio para el estudio de alternativas terapéuticas potenciales contra la malaria. El objetivo de esta revisión es brindar una visión general de los hallazgos frente a las NPP y los estudios realizados en cuanto a los compuestos encontrados que inhiben o disminuyen su actividad.

\section{METODOLOGÍA}

\section{Método de búsqueda de información}

Se diseñó una estrategia de búsqueda, en español e inglés, en las bases de datos electrónicas PubMed y ScienceDirect, y en revistas relacionadas al tema de interés como Blood y Malaria Journal, con las siguientes palabras clave: NPP, Plasmodium, inhibitors, malaria. Además, se realizaron búsquedas secundarias en las listas de referencias bibliográficas de los artículos identificados.

\section{Criterios de inclusión y exclusión de los estudios en la revisión}

Se incluyeron los artículos de investigación y revisión de tema hasta el año 2019 que cumplieran con las palabras clave y que arrojaban información sobre la biología, función e inhibidores de NPP en Plasmodium spp. 


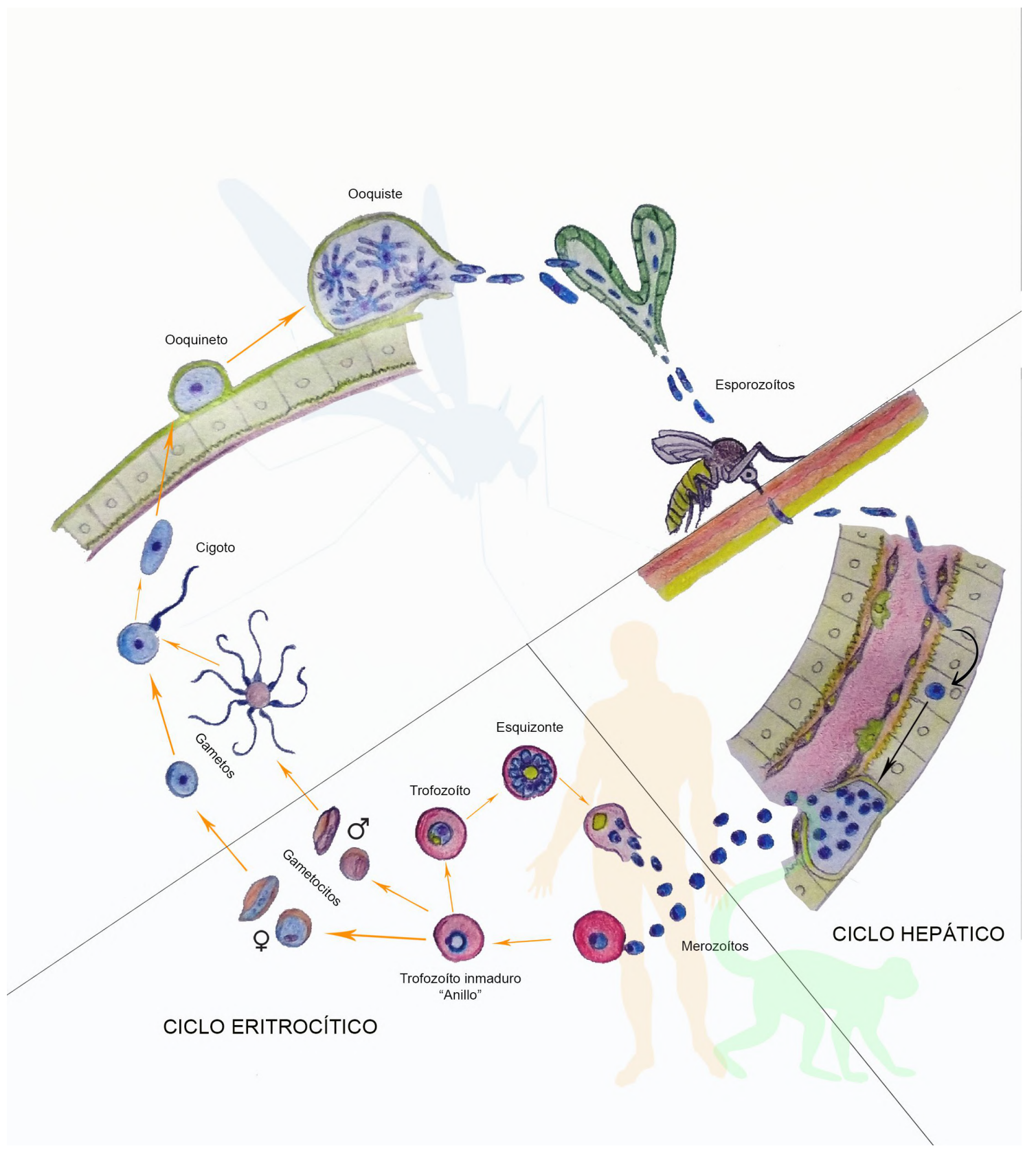

Figura 1. Ciclo de vida de Plasmodium sp. Consiste en una fase sexual, desarrollada en el mosquito hembra del género Anopheles; allí, los gametocitos masculinos y femeninos adquiridos por la picadura a un humano o primate infectado, se diferenciarán en gametos para su posterior fusión y formación del cigoto, que invadirá la pared intestinal del mosquito pasando por ooquineto y luego ooquiste, liberando esporozoítos que invadirán las glándulas salivares. La picadura del mosquito infectado inyecta los esporozoítos en un hospedero humano o primate, allí viajarán por vía sanguínea o linfática hacia el hígado donde iniciará su fase asexual, invadiendo los hepatocitos y formando esquizontes hepáticos, y posteriormente liberando merosomas con cientos de merozoítos a sangre periférica. Estas formas infectantes invadirán eritrocitos y allí se diferenciarán en trofozoítos y por esquizogonia formarán esquizontes que darán liberación a múltiples merozoítos infectantes. Algunos trofozoítos no madurarán hacia esquizonte, sino que se diferenciarán a gametocitos para ser ingeridos por un mosquito y continuar su ciclo. Tomado y adaptado de Bannister y Mitchell, 2003. 
Luego de la elección de los artículos, se excluyeron aquellos cuya metodología y hallazgos no se relacionaban con las palabras clave y el objetivo de la revisión.

\section{RESULTADOS}

Se obtuvo un total de 75 artículos luego de eliminar los repetidos en idioma inglés, de los cuales se seleccionaron 60 por título, materiales y métodos y resumen; de éstos se excluyeron 8 por no presentar resultados de estudios pertinentes al tema. En total, se analizaron 52 referencias. Los principales resultados del análisis de estas lecturas son los siguientes:

\section{Generalidades de la membrana del eritrocito}

El eritrocito posee una membrana celular permeable a una variedad de solutos necesarios para su desarrollo y funcionamiento. Esta membrana está compuesta por una bicapa lipídica con pesos iguales en colesterol y fosfolípidos; se encuentran cuatro tipos de fosfolípidos: esfingomielina, fosfatidilcolina, fosfatidiletanolamina y fosfatidilserina (Mohandas y Gallagher, 2008). Las relaciones lipídicas en la membrana se ven afectadas al momento de infección por el parásito Plasmodium, observándose una disminución en la proporción de esfingomielina $(47 \%)$ y en la relación colesterol/fosfolípidos (55\%), lo cual altera la permeabilidad de la membrana y las funciones sobre sus transportadores (López y Segura, 2008). Debido a que la membrana trabaja como una barrera selectiva para los nutrientes de la célula, es necesario que este proceso esté mediado por proteínas con diversas funcionalidades, como el transporte de iones que conllevan a la regulación del potencial de membrana, la osmolaridad, entre otras. Se han caracterizado alrededor de 50 proteínas transmembranales, y al menos 12 poseen funciones transportadoras, como bombas $\mathrm{Na}^{+} / \mathrm{K}^{+}$, cotransportador de $\mathrm{NaKCl}_{2}$, cotransportador $\mathrm{Na}^{+} / \mathrm{K}^{+}, \mathrm{Ca}^{++}$ATPasa, banda 3 (transporte de iones) (Salhany, 2004), diversas acuaporinas, Glut1 (Blodgett y Carruthers, 2004), antígeno JK (proteína antígeno kidd), RhAg, $\mathrm{K}^{+} \mathrm{Cl}^{-}$cotransportador, $\mathrm{Na}^{+} \mathrm{Cl}^{-}$cotransportador y canal de Gardos (Mohandas y Gallagher, 2008). Cuando hay infección por P. falciparum, el patógeno aprovecha los mecanismos endógenos de la célula huésped y exporta otras proteínas hacia la membrana eritrocitaria para la captación de nutrientes a los que el eritrocito no infectado no es permeable, con el fin de suplir sus necesidades fisiológicas y bioquímicas. Frente a la actividad de los mecanismos de transporte, los reticulocitos o eritrocitos jóvenes poseen una mayor actividad debido a que son células que están finalizando su proceso de maduración, por lo que $P$. vivax tiene preferencia a invadir dichas células; caso contrario sucede con $P$. falciparum, que presenta menos preferencia por invadir reticulocitos e invade eritrocitos de diferentes edades (Kirk, 2001).

\section{Nuevas rutas de permeabilidad (NPP) inducidas por $P$. falciparum}

Como se mencionó anteriormente, el eritrocito es uno de los elementos fundamentales para el desarrollo y la proliferación del parásito. Debido a la ausencia de una gran variedad de nutrientes en el citosol del huésped, el patógeno recurre a un novedoso e ingenioso mecanismo que consiste en modificar la membrana biológica del eritrocito y volverla más permeable a diversos nutrientes y iones, recibiendo el nombre de nuevas rutas de permeabilidad (NPP); además de utilizar algunos sistemas de transporte endógeno del eritrocito para captar solutos necesarios para su óptimo crecimiento. Diversos estudios se han enfocado en determinar el origen y la naturaleza de las NPP, descubriendo nuevas proteínas exportadas por el parásito durante su invasión que aumentan el eflujo e influjo de solutos (Kirk, 2001).

\section{Proteínas implicadas en las NPP}

En vista de que el parásito exporta una gran variedad de proteínas, se acepta el modelo en el cual se utiliza una vía secretora común para las diversas proteínas, tanto las que tienen como finalidad la vacuola parasitófora (PV) como las que necesitan atravesar la membrana de la vacuola parasitófora (PVM) con el objetivo de llegar al citosol y/o membrana eritrocitaria. Mencionando el caso particular de las proteínas que requieren traspasar la PVM, estas, al instaurarse en la membrana eritrocitaria cumplen su función, la cual sería permitir el paso de solutos y conformar las NPP (Gilson et al., 2017) (figura 2). El inicio se da en el retículo endoplásmico (RE), demostrado con el inhibidor de transporte Brefeldin A, el cual impidió 
el transporte de vesículas entre el RE y el aparato de Golgi, quedando retenidas las proteínas marcadas en el RE (Gilson et al., 2017). Debido a que esta ruta es utilizada para gran variedad de proteínas, éstas deben diferenciarse al momento de su empaquetamiento y distribución, por lo que requieren de un motivo de localización denominado PExEL (elemento de exportación de Plasmodium); este motivo permite al parásito realizar una distinción de la finalidad de las proteínas. PExEL consta de cinco residuos de aminoácidos RxLxE (x es cualquier aminoácido no cargado) y se ubica cerca del extremo $\mathrm{N}$ de la carga a exportar, el cual es sintetizado con anterioridad (Gilson et al., 2017). Luego de que el motivo PExEL es agregado a la carga, éste se empaqueta en vesículas y pasa directamente a la PVM, donde se encuentra un complejo encargado del transporte desde la PV hacia el eritrocito, denominado PTEX (translocón de exportación de proteínas de Plasmodium), el cual reconoce dicha secuencia de aminoácidos y permite el paso de la proteína (Elsworth et al., 2014). Frente al descubrimiento de las NPP, diversos autores han investigado el cambio en las proteínas de los eritrocitos, indagando a fondo si son éstas las que aumentan su actividad o si se mantienen inactivas o no tienen implicación alguna frente al excesivo incremento del ingreso de solutos.

\section{NPP y su relación con el transporte de iones}

Dada la necesidad de comprender el mecanismo exacto por el cual el parásito induce un aumento en la permeabilidad del eritrocito, se han realizado estudios basados en técnicas fluorescentes y técnicas electrofisiológicas como el Patch-clamp, que permite cuantificar el ingreso y egreso de iones de algunos

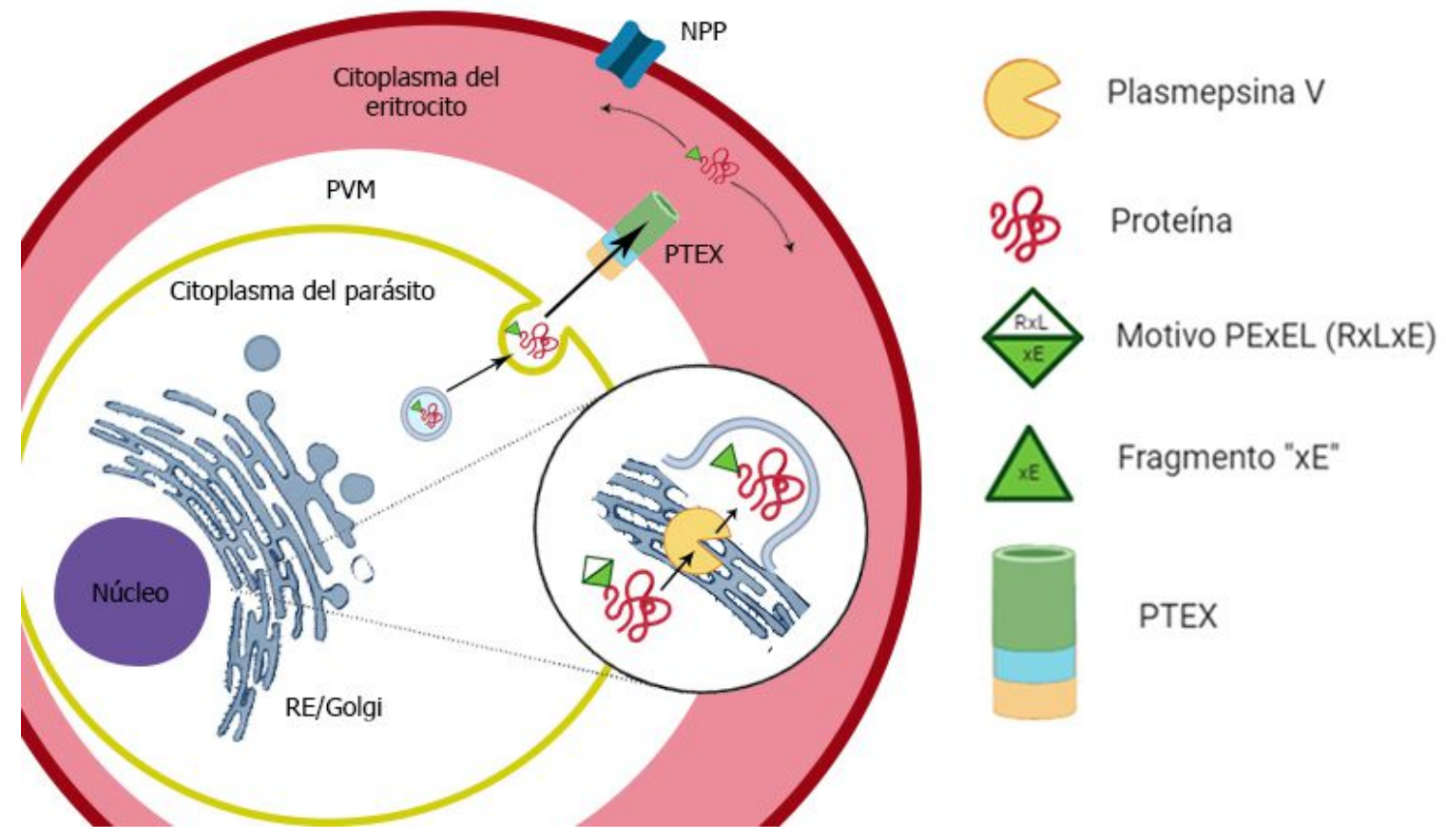

Figura 2. Modelo de exportación de proteínas. Inicia en el Retículo Endoplásmico (RE), en el cual un motivo denominado PExEL que consiste en una serie de aminoácidos RXLXE (donde $x$ es cualquier aminoácido no cargado), se une al extremo $\mathrm{N}$ terminal de la proteína. Con este marcaje, la Plasmepsina $\mathrm{V}$ lo reconoce y escinde el motivo, liberando los aminoácidos RxL, dejando la proteína con el fragmento xE. Posteriormente, se empaqueta en vesículas y sale hacia la membrana de la vacuola parasitófora (PVM), en donde un complejo proteico denominado PTEX reconoce la secuencia y permite el paso de la proteína hacia el citoplasma del eritrocito. Fuente: elaboración de los autores. 
canales en particular. Ginsburg et al. (1983) así como Kirk (2001) proporcionaron información importante sobre la naturaleza de las NPP, mediante estudios realizados con mediciones de flujo y hemólisis; estos trabajos determinaron que las NPP constan de un canal aniónico de alta conductancia y de poca selectividad, que permite el ingreso de distintos iones orgánicos e inorgánicos. A partir de este hallazgo, se denominó a este canal como PSAC (Plasmodial Surface Anion Channel) (Thomas y Lew, 2004; Zipprer et al., 2014). También se ha estudiado el papel de otros canales de conductancia, como los ORC (Outwardly rectifying intermediate conductance anion channel), los cuales pueden presentar una función reguladora de volumen, aunque sólo en presencia de albúmina sérica y se cree es fundamental en el desarrollo del parásito (Huber et al., 2008).

Para comprobar la existencia de PSAC, se han realizado mediciones cuantitativas del flujo de iones en glóbulos rojos parasitados, mediante un inhibidor de PSAC, la furosemida, (Alkhalil et al., 2004). Alkhalil et al. (2004) confirmaron, mediante Patch-clamp, la dependencia de voltaje del ingreso de $\mathrm{Cl}^{-}$a PSAC, así como al lactato y sorbitol, lo que reafirma que las NPP están dadas por un solo canal permeable a distintos solutos. Se han analizado las evidencias propuestas sobre las NPP y se desarrolló un estudio sobre el papel de la fosforilación sobre los canales aniónicos durante la infección por $P$. falciparum; debido a la hipótesis de que el parásito es capaz de inducir la activación de distintos canales inactivos en el eritrocito mediante dos mecanismos, los cuales incluyen el estrés oxidativo y la fosforilación (Merckx et al., 2008), se evaluó la actividad de las vías de señalización dependientes de APMc, por lo tanto se tomó la proteína quinasa A (PKA), la cual es un componente fundamental en dicha vía, para examinar su influencia en la activación del canal. PKA regula la exocitosis de los orgánulos de Plasmodium mediante una vía de señalización dependiente de AMPc, además del ingreso excesivo de $\mathrm{Ca}^{++}$, esta vía se ve activada y aumenta aún más la cantidad de ingreso de $\mathrm{Ca}^{++}$requerido para la exocitosis de componentes del patógeno (Merckx et al., 2008). De igual manera, la adición de PKA y ATP en glóbulos rojos no infectados indujo una corriente en la membrana que era similar a los eritrocitos infectados, sugiriendo también un mecanismo de regulación positiva que involucra la fosforilación (Decherf et al., 2004). Un estudio realizado por Zipprer et al. (2014), confirmó que el aumento de la entrada de $\mathrm{Ca}^{++}$es dado por unas nuevas vías impuestas por el parásito, evaluando con compuestos fluorescentes como el Fluo-8, la cantidad de $\mathrm{Ca}^{++}$en células infectadas y células no infectadas, encontrando una gran diferencia entre ambas células. Por medio de análisis de citometría de flujo, se determinó que unas subpoblaciones de glóbulos rojos inducen una permeabilidad frente al $\mathrm{Ca}^{++}$cuando se someten a estrés mecánico, lo que es característico de canales mecanosensibles y que puede contribuir a la protección contra la malaria al evitar la maduración del parásito dentro del eritrocito (Brain et al., 2004). Así mismo sucede con diversos canales pertenecientes a eritrocitos de perro, en donde son activados por estimulación de contracción y acidificación (Dunham, 2004); sería prudente evaluar estos mecanismos en glóbulos rojos humanos infectados y no infectados.

Debido a que el $\mathrm{K}^{+}$juega un papel importante en la regulación electroquímica y de osmolaridad en las células, se evaluó el estado de los canales transportadores de $\mathrm{K}^{+}$en eritrocitos infectados con $P$. falciparum y su relación con las NPP. Para ello, Waller et al., identificaron dos genes del parásito y su localización durante la infección, PfK1 y PfK2. Los autores hipotetizan que el gen $P f K 1$ codifica para una proteína con función putativa de canal que es exportada hacia la membrana eritrocitaria para el influjo de $\mathrm{K}^{+}$(Waller et al., 2008). Para PfK2 se encontró que está en mayor proporción en merozoítos y en estadios tardíos del parásito, tales como esquizontes; lo que sugiere que $P f K 2$ requiere un mayor tiempo para su expresión, y también se plantea la hipótesis de que $P f K 2$ es un canal exclusivo para el merozoíto. Estos procesos de ingreso de $\mathrm{K}^{+}$son desencadenados por la entrada excesiva de $\mathrm{Ca}^{++}$, por lo que es necesaria la transcripción de genes para el mantenimiento del potencial de membrana (Waller et al., 2008). Kucherenko et al. (2012) analizaron el papel en el incremento de la permeabilidad de la membrana eritrocitaria, evaluando una proteína encargada del paso de distintos solutos y la cual se activa mediante el incremento de AMPc, como lo es la Acuaporina 9 (AQP9). Para evaluar la actividad 
y su relación con el intercambio iónico, se tuvieron dos tipos de ratones, uno silvestre (wild type) que contenía los genes para la AQP9 $(\operatorname{aqp} 9+/+)$ y otro genéticamente modificado que carecía de genes necesarios para la codificación de la AQP9 (aqp-/-). Usando la técnica de patch-clamp hicieron mediciones en distintas soluciones isotónicas para identificar la diferencia de permeabilidades a los distintos iones y encontraron que $\mathrm{K}^{+}$era el de mayor influjo, seguido por el $\mathrm{Na}^{+}$y por último el $\mathrm{Ca}^{++}$. En cuanto a los ratones deficientes de la proteína, éstos presentaron menor ingreso en todos los iones comparado con los wild type. Por lo tanto, los parásitos P. berghei que infectan ratones genéticamente mutados para la AQP9, presentan baja parasitemia comparados con los ratones infectados, pero con presencia de la proteína; esto debido a que los niveles de $\mathrm{Na}^{+}$ y $\mathrm{Ca}^{++}$necesarios para el crecimiento del patógeno intracelular son más bajos (Kucherenko et al., 2012).

\section{Transporte de aminoácidos mediados por las NPP}

Como se mencionó antes, P. falciparum digiere la hemoglobina $(\mathrm{Hb})$ presente en los eritrocitos, aprovechando los aminoácidos allí presentes para la síntesis de sus proteínas y secretando un porcentaje de esos aminoácidos al medio exterior. La isoleucina es un aminoácido esencial para la supervivencia del parásito y la $\mathrm{Hb}$ adulta no la posee, por lo que es necesario adquirirla del medio extracelular (Cobbold et al., 2010). Kirk y colegas analizaron la tasa de permeabilidad a la isoleucina en eritrocitos infectados y encontraron en estos eritrocitos que la tasa inicial aumentó hasta 5 veces comparando con eritrocitos no infectados en condiciones iguales (Martin y Kirk, 2007). El transporte de la isoleucina en eritrocitos sanos es mediado por la vía del sistema L, siendo evaluada su implicación frente al incremento en el ingreso del aminoácido en glóbulos rojos parasitados; para ello utilizaron dos inhibidores, el BCH (inhibitor 2-aminobicyclo-heptane-2-carboxylic acid) el cual es un inhibidor del sistema $\mathrm{L}$ y la furosemida, que se sabe es un inhibidor parcial de las NPP (inhibiendo el transporte de cloro) (López y Segura, 2008). El $\mathrm{BCH}$ redujo notablemente el ingreso del aminoácido en células rojas sanas hasta en un $80 \%$, en cuanto a los eritrocitos infectados no tuvo un mayor efecto en la reducción del influjo de la isoleucina (Martin y Kirk, 2007). Frente al inhibidor furosemida, los eritrocitos sanos no presentaron cambio alguno, en comparación con los infectados que tuvieron una reducción del $80 \%$ en el influjo del aminoácido (Martin y Kirk, 2007). De acuerdo con los resultados obtenidos con la isoleucina, se decidió evaluar el incremento en la permeabilidad frente a la metionina y el rol de las NPP en este aumento. La metionina es otro aminoácido esencial para el parásito, y también comparte el sistema L del eritrocito. De acuerdo con los resultados obtenidos, la permeabilidad y el influjo del aminoácido incrementó drásticamente en células rojas parasitadas comparado con las no parasitadas. Evaluando también el sistema L endógeno y las NPP, se utilizaron los dos mismos inhibidores utilizados con la isoleucina, el BCH (para el sistema L) y la furosemida (para las NPP); encontrando resultados similares a los obtenidos con la isoleucina, confirmando inhibición del $95 \%$ de ingreso de metionina en eritrocitos no infectados y poca eficacia contra los infectados; así mismo, la furosemida inhibió en un $90 \%$ la captación de metionina en células infectadas y no hubo inhibición alguna en no infectados. Esto confirma la presencia de las NPP y su importancia en el transporte de distintos aminoácidos de importancia para Plasmodium (Cobbold et al., 2010).

\section{Transporte de otras moléculas a través de las NPP}

Las nuevas vías inducidas por el parásito generan un microentorno favorable para el parásito, regulando la entrada e incluso favoreciendo la salida de productos de desecho, tal como el ácido láctico. Estudios realizados han dado a conocer que el influjo de monosacáridos aumenta en gran medida frente a la invasión de los eritrocitos, evidenciando incrementos en la entrada y consumo de glucosa mucho mayores que en eritrocitos no infectados, presentándose un consumo de hasta 100 veces mayor (Kirk y Saliba, 2007). La membrana del eritrocito posee un mecanismo para el transporte de glucosa mediado por el GLUT1, el cual es utilizado por el parásito en mayor medida para el ingreso de la glucosa. Para evaluar el rol de GLUT1 y la relación con las NPP, se inhibió el mecanismo endógeno, midiendo los niveles de glucosa mediante las nuevas rutas de permeabilidad, hallando que la vía de las NPP ingresa menos glucosa que la mediada por el GLUT1 del eritrocito, por lo que este trans- 
portador es fundamental para la supervivencia del parásito (Kirk y Saliba, 2007). El sorbitol también presenta un fenómeno similar, evidenciándose con un alto ingreso a las células parasitadas, teniendo una permeabilidad aproximadamente 10 veces mayor que las células no parasitadas (Staines et al., 2006). Para medir la velocidad de penetración del sorbitol, se indujo una hemolisis isosmótica en células normales y células infectadas para una posterior comparación; en este experimento se halló que el sorbitol ingresa casi el doble de rápido en células infectadas (Ginsburg et al., 1983). Estos hallazgos, se evidencian en etapas tardías del parásito, siendo indetectables durante las primeras horas, correspondiente al estadio de anillo y apareciendo en etapas más maduras (Ginsburg et al., 1983). Counihan et al. (2017) evaluaron el efecto que tiene la carencia de la proteína RHopH2 de Plasmodium, la cual forma un complejo con la proteína RHopH1 (una proteína involucrada con la actividad de las NPP), con el transporte del sorbitol; evidenciando reducciones en muchos aspectos del desarrollo parasitario, tales como las transiciones de etapas y el número reducido de merozoítos por esquizonte, además de la privación en la captación de diversos nutrientes en las NPP; esto se dedujo debido a que los parásitos carentes de la RHopH2 tenían una reducción significativa en la lisis por sorbitol. Un estudio realizado en 2004, mediante hemólisis isosmótica, permitió definir la permeabilidad a distintos azucares durante la invasión del eritrocito y se encontró que el sorbitol es el más permeable, siguiéndole el manitol y por último la sacarosa (Duranton et al., 2004). Comparando la permeabilidad frente a la taurina y el sorbitol en dos especies de Plasmodium: P. falciparum y P. gallinaceum (en pollos), se encontró que el primero es más sensible a los inhibidores furosemida y NPPB que $P$. gallinaceum; hallando también una preferencia de $P$. falciparum frente a la taurina sobre el sorbitol, siendo lo contrario para P. gallinaceum (Staines et al., 2001).

Además de la cantidad de solutos descritos anteriormente, también se le ha otorgado la responsabilidad del transporte de otras moléculas como las vitaminas y precursores de fosfolípidos. El ácido pantoténico (vitamina B5) o en condiciones fisiológicas como pantotenato, cumple diversas funciones en el parásito, una de ellas funcionando como cofactor de la coenzima A ( CoA), por lo que el parásito lo requiere para su desarrollo (Kirk y Saliba, 2007). El pantotenato ingresa de una forma nula o muy lenta por membrana eritrocitaria, por lo que el parásito se ayuda a través de la implementación de las NPP (Kirk y Saliba, 2007). En cuanto a la colina, se ha descubierto que posee una permeabilidad aproximadamente 100 veces mayor que la del sorbitol (Staines et al., 2006). Este nutriente es esencial para el parásito porque permite la formación de fosfolípidos como la fosfatidilcolina para el cambio en la composición lipídica en la membrana eritrocitaria. In vitro, se muestra un incremento en la afluencia de colina en eritrocitos infectados con $P$. falciparum, especulando que las concentraciones fisiológicas de la colina son mediadas por transportadores endógenos mejorados (en aproximadamente $2 / 3$ ) y por medio de las NPP (en $1 / 3$ ) (Kirk y Saliba, 2007). Se ha descubierto que las NPP posen dos conductancias aniónicas, una hacia el interior y otra hacia el exterior. La conductancia hacia el exterior se le ha atribuido a la participación en el transporte de aminoácidos, osmolitos orgánicos y de productos de desecho, como el lactato (Duranton et al., 2004).

\section{Rol del CLAG3 en la permeabilidad}

Hasta el momento se desconoce la participación de la proteína CLAG3 en las NPP (Counihan et al., 2017), por lo que se expondrán algunos descubrimientos e hipótesis frente su posible rol. En 2011, se descubrió la participación de los genes clag, mediante unas mutaciones puntuales, y otorgándole a la proteína CLAG3 una interacción con una o más proteínas que contribuían a la formación de PSAC (Nguitragool et al., 2011). Sumado a esto se le otorgó un consistente papel de dos genes de clag3 al transporte mediado por PSAC (Bowlin et al., 2012). Un estudio reciente apoya la participación de la proteína CLAG3 en la formación de PSAC; debido a que esta es integral en la membrana eritrocitaria y posee motivos anfipáticos, y también forma complejos con RhopH2 y RhopH3 (High molecular mass protein complex in the rhoptries), contribuyendo a la formación de poros, teniendo así una acción como modulador de la captación de solutos (Gupta et al., 2018). Aunque se conoce la participación de RhopH2 y RhopH3 en 
la formación de PSAC, aún se no dilucida su función concreta, pudiendo actuar como formadora de complejos con CLAG3 o en el transporte de CLAG3, actuando como chaperonas (Zainabadi, 2016). Zainabadi (2016) descubrió tres complejos que implicaban a CLAG3, de tamaños variables $(480 \mathrm{kDa}, 620 \mathrm{kDa}$ y $700 \mathrm{kDa}$ ), en los que se especula que son formados por varias proteínas CLAG3 y las RhopH2/3. Si bien se asegura la contribución de CLAG3 en las NPP, aún se desconoce su mecanismo exacto y la participación de otras proteínas en la formación de los complejos.

\section{Compuestos inhibidores de las NPP}

La presencia de las NPP atrajo la atención de varios investigadores como un posible blanco terapéutico, lo que conllevó a la realización de diversos estudios en donde se evaluó la actividad de compuestos y su viabilidad como futuros fármacos. Hasta el momento se han caracterizado una gran cantidad de compuestos y su posible mecanismo de acción en las nuevas rutas de permeabilidad, entre los más conocidos y usados, se encuentra la furosemida (Alkhalil et al., 2004; Dickerman et al., 2016) y el 5-nitro-2-3fenilpropilaminobenzoico (NPPB) (Dickerman et al., 2016; Lisk et al., 2006; Gilson et al., 2016). Estudios que han intentado descifrar el mecanismo de acción preciso de la furosemida proponen que éste inhibe a PSAC ingresando al poro y taponándolo; aunque por medio de un experimento en el cual se medían las distribuciones de tiempo en el cual el poro de PSAC se mantenía cerrado, se halló que no había un cambio significativo en presencia o ausencia del inhibidor, generando como conclusión que la furosemida puede actuar como un inhibidor alostérico (Alkhalil et al., 2004). En cuanto a NPPB no se ha asociado a un mecanismo específico que describa la manera en cómo inhibe el transporte de diversos solutos a través de las NPP.

Debido a que los inhibidores existentes hacia PSAC son altamente inespecíficos, éstos pueden afectar e inhibir canales propios (Lisk et al., 2006). Ante esto se ha buscado un compuesto que inhiba el transporte mediado por PSAC y que no posea efectos negativos. Al Dantroleno, que es un relajante muscular que inhibe la liberación de $\mathrm{Ca}^{++}$en el $\mathrm{RE}$, se le evaluó su eficacia y su mecanismo de acción relacionado con la inhibición de las NPP en P. falciparum, encontrando que éste produce una disminución en la lisis inducida por sorbitol, atribuyéndole también una inhibición de la permeabilidad frente a distintas moléculas. En cuanto a su mecanismo de acción, se explica que éste posee una interacción con la cara extracelular de PSAC, lo cual influye directamente en la apertura del canal (Lisk et al., 2006). Compuestos biotinizados (Sulfo-NHS-biotin) que son permeables en el eritrocito después de la invasión del parásito, tienen la capacidad de bloquear irreversiblemente las vías, produciendo una reducción en el desarrollo de los parásitos (Baumeister et al., 2003). La longitud de las cadenas de carbono en los inhibidores de Sulfo-NHS y Sulfo-NHS-biotin tiene una implicación en el bloqueo del canal, encontrándose que entre más largo sea el brazo, más reduce el ingreso de los solutos por medio de esta vía (Cohn et al., 2003). La biotinización se ha reportado como inhibidor en otros transportadores, como el PfENT1, para el cual el MTS-etil-amoniobiotina tiene capacidad inhibitoria (Nishtala et al., 2018).

Muchos compuestos se han evaluado para determinar su potencial como antimalárico e inhibidor de las nuevas rutas de permeabilidad. A distintos derivados del ácido cinámico se les evaluó su actividad en el crecimiento del parásito, encontrando que todos son potentes inhibidores en la captación de sorbitol, teniendo al UK-5099 como el más potente, con una concentración inhibitoria $50\left(\mathrm{IC}_{50}\right)$ de $0.052 \mathrm{mM}$ contra estadíos inmaduros (Kanaani y Ginsburg, 1991). Un estudio realizado por Dickerman y colaboradores, demostró que dos compuestos extraídos de la Malaria Box (MMV007571 y MMV020439) poseen una buena actividad contra $P$. falciparum, evidenciándose con un $\mathrm{IC}_{50}$ de $367 \mathrm{nM}$ para MMV007571 y $222 \mathrm{nM}$ para MMV020439, inhibiendo las NPP y la enzima DHODH, siendo unos potenciales inhibidores (Dickerman et al., 2016). La Deferoxamina (DFO) posee actividad antimalárica tanto in vitro como in vivo, para lo que se propuso generar un compuesto hibrido entre la DFO y el metil antranilato (MA) y comparar la actividad entre ambos compuestos; hallando que el compuesto híbrido, MA-DFO, es mucho más eficaz que el DFO, con un $\mathrm{IC}_{50}$ de $3 \pm 1 \mu \mathrm{M}$ y $30 \pm 8 \mu \mathrm{M}$, respectivamente; teniendo una mayor velocidad de acción $\mathrm{IC}_{50} 10$ veces menor y actividad en etapas 
maduras y tempranas (Loyevsky et al., 1993). Otros compuestos como la bafilomicina A1 y el protonóforo FCCP redujeron el transporte de colina, alcanzando a reducir el ingreso de dicho soluto hasta en un $50 \%$ (Biagini et al., 2004). Teniendo un efecto similar con el transporte de colina se encuentra la NEM (Netilmaleimida) (Philippot et al., 1991). Staines et al. (2004) evaluaron el efecto de la Mefloquina sobre las NPP, encontrando resultados nulos. Los autores concluyeron que este fármaco no tiene actividad sobre el transporte de nutrientes en el parásito.

Las diaminidas poseen un potencial de inhibición en los glóbulos rojos parasitados, exhibiendo una actividad antimalárica in vitro importante contra distintas cepas, con un $\mathrm{IC}_{50}$ de $66 \mathrm{mM}$ contra $P$. falciparum K1. La pentamidina, tiene una rápida captación mediada posiblemente por las nuevas vías de permeabilidad, ya que la captación de este soluto se inhibe por la furosemida. Otro mecanismo propuesto para este compuesto es su acción sobre la ferriprotoporfina IX (FPIX), inhibiendo la formación de hemozoína y formación de complejos tóxicos para el parásito (Bray et al., 2001).

Los arilaminobenzoatos como el NPPB, son una fuente de compuestos que pueden ser útiles para la fabricación de nuevos fármacos contra la malaria. Para evaluar el efecto de diversos arilaminobenzoatos, se midió su capacidad de inhibición del transporte de distintos solutos en $P$. falciparum, hallando cuatro compuestos (NPPB, NPBB, MENB y NDPB) altamente potentes contra el transporte de colina, timidina y lactato (Kirk y Horner, 1995). NDPB mostró poco efecto en proteínas implicadas en el transporte de $\mathrm{Cl}^{-}$en el riñón y tampoco se evidenció efecto en la proteína banda 3 del eritrocito. Sumado a esto, muchos de los compuestos actualmente utilizados para la inhibición de las NPP, pierden o disminuyen su efecto en presencia de suero (Lisk et al., 2006), pero NDPB demostró que, aunque no es el inhibidor más potente, no se ve afectado en presencia de suero, por lo que promete ser un compuesto útil y merece un estudio más a fondo (Kirk y Horner, 1995).

Otro blanco terapéutico que está directamente implicado en la formación de las nuevas rutas de permeabilidad es la Plasmepsina V, la cual tiene una función clave en la escisión de proteínas con destino hacia la membrana eritrocitaria, como sucede con el motivo PExEL en el RE (Boddey et al., 2013). Existen unos compuestos peptidomiméticos que pueden inhibir potentemente la Plasmepsina V, bloqueando así la exportación de las proteínas y posteriormente disminuyendo el crecimiento del parásito. La mayoría de estos peptidomiméticos poseen una Arg en la posición P3, lo que indica la importancia del aminoácido para la actividad; la evaluación de diversos compuestos sintéticos en donde existe otra molécula en la posición P3, permitió encontrar que la Caravanina es un reemplazo ideal para la Arg, teniendo un potente efecto contra la Plasmepsina V (Gazdik et al., 2016).

\section{Nuevas consideraciones}

Aún falta mucho por comprender acerca de las NPP y de fármacos que puedan ser útiles para el tratamiento de la malaria, en especial que actúen frente a este blanco. Es necesario considerar y tener en cuenta estudios previos de inhibidores sobre canales en otras células que puedan servir como punto de partida para encontrar un fármaco potencial contra el Plasmodium; como el Bretilio, un antiarrítmico que funciona sobre la bomba de $\mathrm{Na}^{+}$, comportándose como $\mathrm{K}^{+}$e inhibiendo la bomba, quedando atrapado en la conformación E2 P/ catión (Helms et al., 2004); por lo que sería un indicio para sustancias derivadas de éste y con posible acción sobre PSAC. Así mismo, indagar más sobre las proteínas y genes implicados en todo el proceso de las NPP, como la inhibición de la Plasmepsina $\mathrm{V}$, que al caracterizarse bien la posición de sus aminoácidos esenciales, tal como la Arg en P3, puede ser un gran indicio para nuevas clases de inhibidores (Taglialatela et al., 2015). Además, sería valioso realizar una comparación de exportomas entre especies de Plasmodium y otros parásitos pertenecientes al Filo Apicomplexa, tal como Babesia o Toxoplasma, lo que permitiría identificar las diferencias en las proteínas implicadas en el transporte y su posible origen (Baumeister et al., 2015).

En conclusión, las nuevas rutas de permeabilidad son un blanco terapéutico valioso, debido a la importante participación en el influjo/eflujo de una gran cantidad de solutos necesarios para el desarrollo y supervivencia del parásito. Se han hecho avances 
significativos frente a la caracterización y funciones, pero aún quedan muchos vacíos en torno a los genes y proteínas involucradas, lo cual genera preguntas que aporten al conocimiento y al descubrimiento de nuevos compuestos eficaces y selectivos frente a las NPP de P. falciparum, por ejemplo: ¿Cuál es la similitud química y molecular entre los compuestos que bloquean estas rutas? ¿Qué proteínas aún no se han identificado dentro de este complejo? ¿Cuál sería la bioactividad de compuestos derivados de microorganismos o plantas frente a las NPP? ¿Cómo funcionan estas vías en las diferentes especies de Plasmodium?

\section{AGRADECIMIENTOS}

Yeison Castañeda fue acreedor de un estímulo como Joven investigador del CODI adscrito al Grupo Malaria de la Universidad de Antioquia.

\section{CONFLICTO DE INTERESES}

Los autores declaran no tener conflictos de intereses.

\section{REFERENCIAS}

Alkhalil, A., Cohn, J., Wagner, M., Cabrera, J., Rajapandi, T., \& Desai, S. (2004). Plasmodium falciparum likely encodes the principal anion channel on infected human erythrocytes. Blood Journal, 104(13), 4279-86. DOI:10.1182/blood-2004-05-2047

Bannister, L., \& Mitchell, G. (2003). The ins, outs, and roundabouts of malaria. Trends in Parasitology, 19(5), 209-13. DOI:10.1016/s1471-4922(03)00086-2

Baumeister, S., Endermann, T., Charpian, S., Nyalwidhe, J., Huber, S., Lingelbach, K., Lang, F., \& Kirk, K. (2003). A biotin derivative blocks parasite induced novel permeation pathways in Plasmodium falciparum-infected erythrocytes. Molecular and Biochemical Parasitology, 132(1), 35-45. DOI:10.1016/j.molbiopara.2003.08.003

Baumeister, S., Gangopadhyay, P., Repnik, U., \& Lingelbach, K. (2015). Novel insights into red blood cell physiology using parasites as tools. European Journal of Cell Biology, 94(7-9), 332-339. DOI:10.1016/j.ejcb.2015.05.007

Biagini, G. A., Pasini, E. M., Hughes, R., Koning, H. P. De, Vial, H. J., Ward, S. A., \& Bray, P. G. (2004). Characterization of the choline carrier of Plasmodium falciparum: a route for the selective delivery of novel antimalarial drugs. Blood, 104(10), 3372-3377. DOI:10.1182/blood2004-03-1084.Supported

Blodgett, D. M., \& Carruthers, A. (2004). Conventional transport assays underestimate sugar transport rates in hu- man red cells. Blood Cells, Molecules, and Diseases, 32(3), 401-407. DOI:10.1016/j.bcmd.2004.01.015

Boddey, J. A., Carvalho, T. G., Hodder, A. N., Sargeant, T. J., Sleebs, B. E., Marapana, D., Lopaticki, S., Nebl, T., \& Cowman, A. F. (2013). Role of plasmepsin V in export of diverse protein families from the Plasmodium falciparum exportome. Traffic, 14(5), 532-550. DOI:10.1111/tra. 12053

Bowlin, T. L., Lyko, B., Nguyen, S. T., Peet, N. P., Butler, M. M., Dolinta, K., \& Desai, S. A. (2012). Solute restriction reveals an essential role for clag3-associated channels in malaria parasite nutrient acquisition. Molecular Pharmacology, 82(6), 1104-1114. DOI:10.1124/mol.112.081224

Brain, M. C., Pihl, C., Robertson, L., \& Brown, C. B. (2004). Evidence for a mechanosensitive calcium influx into red cells. Blood Cells, Molecules, and Diseases, 32(3), 349352. DOI:10.1016/j.bcmd.2004.01.005

Bray, P. G., DeKoning, H. P., Stocks, P. A., Stead, A. M. W., Edwards, I. G., Elford, B. C., \& Ward, S. A. (2001). Diamidine compounds: Selective uptake and targeting in Plasmodium falciparum. Molecular Pharmacology, 59(5), 1298-1306. DOI:10.1124/mol.59.5.1298

Cabantchik, Z. I., Shanzer, A., Mester, B., Libman, J., Loyevsky, M., \& Lytton, S. D. (1993). The antimalarial action of deferral involves a direct access route to erythrocytic (Plasmodium falciparum) parasites. Journal of Clinical Investigation, 91(1), 218-224. DOI:10.1172/jci116174

Chassaigne, J. A. (2001). Malaria y fármacos antimaláricos. Revista de la Sociedad Venezolana de Microbiología, 21 (2), 85-88. http://ve.scielo.org/scielo.php?script=sci arttext\&pid $=$ S1315-25562001000200017

Cobbold, S. A., Martin, R. E., \& Kirk, K. (2010). Methionine transport in the malaria parasite Plasmodium falciparum. International Journal for Parasitology, 41(1), 125-135. DOI:10.1016/j.ijpara.2010.09.001

Cohn J. V., Alkhalil A., Wagner M. A., Rajapandi T., \& Desai S. A. (2003). Extracellular lysines on the plasmodial surface anion channel involved in $\mathrm{Na}^{+}$exclusion. Molecular and Biochemical Parasitology, 132(1), 27-34. DOI:10.1016/j.molbiopara.2003.08.001

Counihan, N. A., Chisholm, S. A., Bullen, H. E., Srivastava, A., Sanders, P. R., Jonsdottir, T. K., Weiss, G. E., Ghosh, S., Crabb, B.S., Creek, D. J., Gilson, P. R., De Koning-Ward, T. F. (2017). Plasmodium falciparum parasites deploy RhopH2 into the host erythrocyte to obtain nutrients, grow and replicate. ELife, 6, 1-31. DOI:10.7554/eLife.23217

Decherf, G., Egée, S., Staines, H. M., Ellory, J. C., \& Thomas, S. L. (2004). Anionic channels in malaria-infected human red blood cells. Blood Cells, Molecules, and Diseases, 32(3), 366-371. DOI:10.1016/j.bcmd.2004.01.008

Dunham, P. B. (2004). Cell shrinkage activates $\mathrm{Na}^{+} / \mathrm{H}^{+}$ exchange in dog red cells by relieving inhibition of exchange by $\mathrm{Na}^{+}$in isotonic medium. Blood Cells, Molecules, and Diseases, 32(3), 389-393. DOI:10.1016/j.bcmd.2004.01.012

Duranton, C., Huber, S. M., Tanneur, V., Brand, V. B., Akkaya, C., Shumilina, E. V., Lang, F. (2004). Organic osmolyte permeabilities of the Malaria - induced anion conductances in human erythrocytes. The Journal of General 
Physiology, 123(4), 417-426. DOI:10.1085/jgp.200308919

Elsworth, B., Matthews, K., Nie, C. Q., Kalanon, M., Charnaud, S. C., Sanders, P. R., De Koning-Ward, T. F. (2014). PTEX is an essential nexus for protein export in malaria parasites. Nature, 511(7511), 587-591. DOI:10.1038/nature13555

Gazdik, M., Jarman, K. E., ONeill, M. T., Hodder, A. N., Lowes, K. N., Jousset Sabroux, H., Sleebs, B. E. (2016). Exploration of the P3 region of PEXEL peptidomimetics leads to a potent inhibitor of the Plasmodium protease, plasmepsin V. Bioorganic and Medicinal Chemistry, 24(9), 1993-2010. DOI:10.1016/j.bmc.2016.03.027

Gilson, P. R., Chisholm, S. A., Crabb, B. S., \& de KoningWard, T. F. (2017). Host cell remodelling in malaria parasites: a new pool of potential drug targets. International Journal for Parasitology, 47(2-3), 119-127. DOI:10.1016/j.ijpara.2016.06.001

Gilson, P. R., Cobbold, S. A., Elsworth, B., Crabb, B. S., Nie, C. Q., McConville, M. J., \& Dickerman, B. K. (2016). Identification of inhibitors that dually target the new permeability pathway and dihydroorotate dehydrogenase in the blood stage of Plasmodium falciparum. Scientific Reports, 6(1), 1-15. DOI:10.1038/srep37502

Ginsburg, H., Krugliak, M., Eidelman, O., \& Cabantchik, Z. I. (1983). New permeability pathways induced in membranes of Plasmodium falciparum infected erythrocytes. Molecular and Biochemical Parasitology, 8(2), 177-190. DOI:10.1016/0166-6851(83)90008-7

Gupta, A., Balabaskaran-nina, P., Nguitragool, W., Saggu, G. S., Schureck, M. A., \& Desai, A. (2018). CLAG3 Self-Associates in Malaria Parasites and Quantitatively Determines Nutrient Uptake Channels at the Host Membrane. mBio, 9(3), 1-18. DOI:10.1128/mBio.02293-17

Helms, J. B., Arnett, K. L., Gatto, C., \& Milanick, M. A. (2004). Bretylium, an organic quaternary amine, inhibits the Na, K-ATPase by binding to the extracellular Ksite. Blood Cells, Molecules, and Diseases, 32(3), 394-400. DOI:10.1016/j.bcmd.2004.01.013

Huber, S. M., Lang, C., Lang, F., \& Duranton, C. (2008). Organic osmolyte channels in malaria-infected erythrocytes. Biochemical and Biophysical Research Communications, 376 (3), 514-518. DOI:10.1016/j.bbrc.2008.09.036

Instituto Nacional de Salud. (2019). Boletín Epidemiológico Semanal: Semana epidemiológica 52. ISSN 2357-6189. DOI:10.33610/23576189.2019.52

Kanaani, J., \& Ginsburg, H. (1991). Effects of cinnamic acid derivatives on in vitro growth of Plasmodium falciparum and on the permeability of the membrane of malaria-infected erythrocytes. Antimicrobial Agents and Chemotherapy, 36(5), 1102-1108. DOI:10.1128/AAC.36.5.1102

Kirk, K. (2001). Membrane transport in the malaria-infected erythrocyte. Comparative Biochemistry and Physiology Part B: Biochemistry and Molecular Biology, 126(2), S65. DOI:10.1016/S0305-0491(00)80128-5

Kirk, K., \& Horner, H. A. (1995). In search of a selective inhibitor of the induced transport of small solutes in Plasmodium falciparum -infected erythrocytes: Effects of arylaminobenzoates. Biochemical Journal, 311(3), 761768. DOI:10.1042/bj3110761
Kirk, K., \& Saliba, K. J. (2007). Targeting nutrient uptake mechanisms in Plasmodium. Current Drug Targets, 8, 7588. DOI: $10.2174 / 138945007779315560$

Kucherenko, Y. V., Huber, S. M., Nielsen, S., \& Lang, F. (2012). Decreased redox-sensitive erythrocyte cation channel activity in aquaporin 9-deficient mice. Journal of Membrane Biology, 245(12), 797-805. DOI:10.1007/s00232-012-9482-y

Lisk, G., Kang, M., Cohn, J. V., \& Desai, S. A. (2006). Specific inhibition of the plasmodial surface anion channel by Dantrolene. Eukaryotic Cell, 5(11), 1882-1893. DOI:10.1128/ec.00212-06

López, L., \& Segura, C. (2008). Nuevas vías de permeabilidad y regulación del $\mathrm{pH}$ intracelular como posibles blancos terapéuticos en Plasmodium falciparum. Acta Biológica Colombiana, 13(2), 3-22. https://revistas.unal.edu.co/ index.php/actabiol/article/view/1530/2185::pdf

Martin, R. E., \& Kirk, K. (2007). Transport of the essential nutrient isoleucine in human erythrocytes infected with the malaria parasite Plasmodium falciparum. Blood, 109(5), 2217-2224. DOI:10.1182/blood-2005-11-026963

Martin, R. E., Shafik, S. H., \& Richards, S. N. (2018). Mechanisms of resistance to the partner drugs of artemisinin in the malaria parasite. Current Opinion in Pharmacology, 42, 71-80. DOI:10.1016/j.coph.2018.07.010

Merckx, A., Bouyer, G., Thomas, S. L. Y., Langsley, G., \& Egée, S. (2008). Anion channels in Plasmodium-falciparum-infected erythrocytes and protein kinase A. Trends in Parasitology, 25(3), 139-144. DOI:10.1016/j.pt.2008.12.005

Mohandas, N., \& Gallagher, P. G. (2008). Red cell membrane: past, present, and future. Blood, 11(10), 3939-3948. DOI:10.1182/blood-2008-07-161166

Nguitrangool, W., Bokhari, A., Pillai A., Rayavara K., Sharma P., Turpin B, Aravind, L., \& Desai, S. A. (2011). Malaria parasite clag genes determine nutrient uptake channel activity on infected red blood cells. Cell, 145(5), 665-77. DOI:10.1016/j.cell.2011.05.002

Nishtala, S. N., Arora, A., Reyes, J., \& Akabas, M. H. (2018). Accessibility of substituted cysteines in TM2 and TM10 transmembrane segments in the Plasmodium falciparum equilibrate nucleoside transporter PfENT1. Journal of Biological Chemistry, 294(6), 1924-1935. DOI:10.1074/jbc.ra118.006547

Philippot, J. R., Ancelin, M. L., Vial, H. J., Thuet, M. J., \& Parant, M. (1991). Increased permeability to choline in simian erythrocytes after Plasmodium knowlesi infection. Biochemical Journal, 273(3), 701-709. DOI:10.1042/bj2730701

Prudêncio, M., Rodriguez, A., \& Mota, M. M. (2006). The silent path to thousands of merozoites: The Plasmodium liver stage. Nature Reviews Microbiology, 4(11), 849-856. DOI:10.1038/nrmicro1529

Salhany, J. M. (2004). Slow transitions between two conformational states of band 3 (AE1) modulate divalent anion transport and DBDS binding to a second site on band 3 which is activated by lowering the $\mathrm{pH}$ ( $\mathrm{pK} 5.0$ ). Blood Cells, Molecules, and Diseases, 32(3), 372-378. DOI:10.1016/j.bcmd.2004.01.009

Staines, H. M., Godfrey, E. M., Lapaix, F., Egee, S., Tho- 
mas, S., \& Ellory, J. C. (2001). Two functionally distinct organic osmolyte pathways in Plasmodium gallinaceum-infected chicken red blood cells. Biochimica et Biophysica Acta - Biomembranes, 1561(1), 98-108. DOI:10.1016/S0005-2736(01)00461-8

Staines, H. M., Dee, B. C., Shen, M. R., \& Ellory, J. C. (2004). The effect of mefloquine and volume-regulated anion channel inhibitors on induced transport in Plasmodium falciparum-infected human red blood cells. Blood Cells, Molecules, and Diseases, 32(3), 344-348. DOI:10.1016/j.bcmd.2004.01.004

Staines, H. M., Staines, H. M., Ashmore, S., Ashmore, S., Felgate, H., Felgate, H., \& Ellory, J. C. (2006). Solute transport via the new permeability pathways in Plasmodium falciparum-infected human red blood cells is not consistent with a simple single-channel model. Blood, 108(9), 31873194. DOI:10.1182/blood-2006-02-001693.Supported

Taglialatela-Scafati, O., Pedretti, A., Galli, C., Read, M., Pancotti, A., Russo, I., \& Giurisato, E. (2015). Picomolar inhibition of Plasmepsin V, an essential malaria protease, achieved exploiting the prime region. Plos One, 10(11), e0142509. DOI:10.1371/journal.pone.0142509

Thomas, S. L. Y., \& Lew, V. L. (2004). Plasmodium falciparum and the permeation pathway of the host red blood cell. Trends in Parasitology, 20(3), 122-125. DOI:10.1016/j.jqsrt.2018.04.019

Waller, K. L., McBride, S. M., Kim, K., \& McDonald, T. V. (2008). Characterization of two putative potassium channels in Plasmodium falciparum. Malaria Journal, 7, 1-11. DOI:10.1186/1475-2875-7-19

World malaria report 2020: 20 years of global progress and challenges. Geneva: World Health Organization; 2020. Licence: CC BY-NC-SA 3.0 IGO.

Zainabadi, K. (2016). Malaria parasite clag3, a protein linked to nutrient channels, participates in high molecular weight membrane-associated complexes in the infected erythrocyte. PLoS ONE, 11(6), 1-15. DOI:10.1371/journal.pone.0157390

Zipprer, E. M., Neggers, M., Kushwaha, A., Rayavara K., \& Desai, S. A. (2014). A kinetic fluorescence assay reveals unusual features of $\mathrm{Ca}^{++}$uptake in Plasmodium falciparum-infected erythrocytes. Lipids in Health and Disease, 13(1), 1-11. DOI:10.1186/1475-2875-13-184 